\title{
COMPARATIVO DA PROPRIEDADE HV ENTRE AMOSTRAS LAMINADAS CONVENCIONALMENTE E POR MEIO DE LAMINAÇÃO ASSIMÉTRICA*
}

\author{
Andrey de Moraes Barcelos Casanova ${ }^{1}$ \\ Saulo Brinco Diniz² \\ Carolline Serafim da Silva ${ }^{3}$ \\ Talita Gama de Souza ${ }^{4}$ \\ Luiz Paulo Mendonça Brandão ${ }^{5}$ \\ Andersan dos Santos Paula ${ }^{6}$
}

\section{Resumo}

Sendo o alumínio um metal amplamente empregado na produção de partes integrantes de aeronaves, aonde este deve atender a requisitos básicos necessários, como uma boa resistência mecânica, fadiga e corrosão, aliado a baixa densidade, o estudo deste metal se torna pertinente. Visto tais necessidades deste setor, a avaliação destas se torna importante diante da possibilidade de utilização de processos de deformação plástica severa (DPS) para o refinamento de microestrutura, a qual irá acarretará assim, melhoras em propriedades mecânicas. Este trabalho teve por objetivo avaliar o comportamento mecânico, através da dureza HV, medida por um ultramicrodurômetro instrumentado, desejando encontrar uma maior homogeneidade microestrutural bem como o seu refino.

Palavras-chave: Laminação Convencional; Laminação Assimétrica; DPS)

\section{COMPARISON OF HV PROPERTY BETWEEN CONVENTIONAL ROLLING SAMPLES AND ASYMMETRICAL ROLLING}

\section{Abstract}

As aluminum is a metal widely used in the production of integral parts of aircraft, where it is necessary basic requirements, such as good mechanical strength, fatigue and corrosion, together with low density. Therefore the study of this metal becomes pertinent. Considering these needs of this sector, the evaluation of these becomes important in view of the possibility of using severe plastic deformation (DPS) processes for the microstructure refinement, which will lead to improvements in mechanical properties. The objective of this work was to evaluate the mechanical behavior, through the HV hardness, measured by an instrumented ultramicrodrometer, wishing to find a greater microstructural homogeneity as well as refining.

Keywords: Conventional Rolling; Asymmetric Rolling; SPD.

1 M.Sc., Tecnólogo em Processos Metalúrgicos, Doutorando do PGCM, SE-4, IME, Rio de Janeiro - RJ, Brasil.

2 M.Sc., Eng ${ }^{\circ}$. Metalurgista, Doutorando do PGCM, SE-4, IME, Rio de Janeiro - RJ, Brasil; Professor DI - 1, CEFET - Angra dos Reis, Angra dos Reis, RJ, Brasil.

3 M.Sc., Eng ${ }^{a}$. Metalurgista, Analista da Garantia da Qualidade Jr., Galvasud, Porto Real, RJ, Brasil.

$4 \quad$ M.Sc., Química, Doutoranda do PGCM, SE-4, IME, Rio de Janeiro, RJ, Brasil.

5 D.Sc., M.Sc., Engo Metalurgista, Professor Associado do PGCM e da Graduação, SE-4, IME, Rio de Janeiro. RJ, Brasil.

6 D.Sc., M.Sc., Enga Metalurgista, Professora Adjunta do PGCM e da Graduação, SE-4, IME, Rio de Janeiro. RJ, Brasil. 


\section{INTRODUÇÃO}

Devido a setores como o aeronáutico e automobilístico, os quais necessitam de materiais que sejam capazes de atender a demanda de produção, oferecendo características específicas como por exemplo, baixo peso e boa resistência mecânica, o alumínio vem se destacando progressivamente. Graças à significativa resistência mecânica, fadiga e corrosão, associada a baixa densidade, ligas de alumínio como as das séries $2 \mathrm{XXX}$ e $7 \mathrm{XXX}$, estão sendo largamente aplicadas para a manufatura de componentes aeronáuticos (ASM HANDBOOK, 1990) [1].

No que se refere a indústria aeronáutica, para a fabricação de diversos componentes, que por vezes, possuem geometrias complexas, é preciso a utilização de processos de conformação superplástica. Tais processos inferem ao material a propensão de desenvolver valores apreciáveis de deformação plástica (acima de $400 \%$ ) antecedentes a fratura (NIEH, 1997; NIEH, 2005) [2] [3].

A superplasticidade, em geral, requer características como: tamanho de grão em escala nanométrica e meios que visem o impedimento de crescimento de grão durante processos de tratamento térmico e na própria conformação.

Processos de conformação capazes de inferir uma redução no tamanho de grão estão sendo amplamente desenvolvidos e aperfeiçoados. Tais processos, ao passo que refinam a microestrutura, são propícios a elevar a resistência mecânica e tenacidade em determinados materiais. Uma opção viável é a deformação plástica severa (DPS) a qual é formada por diferentes tipos de conformação mecânica, e que possibilita a diminuição de tamanho de grão para fino ou ultrafino, podendo assim, melhorar propriedades físicas (VALIEV, 1993) (ZUO, 2008) [4] [5]. Onde a laminação assimétrica (LA) é um exemplo da DPS.

A laminação é um processo de conformação mecânica no qual é possível reduzir a seção transversal por meio de uma força de compressão. O material a ser laminado é imposto mecanicamente entre dois cilindros de aço ou ferro fundido paralelos e que possuem giro em torno do próprio centro, com velocidade controlada. O produto final, quando se refere ao alumínio, é obtido com espessuras que podem variar desde $150 \mathrm{~mm}$ para chapas grossas até $0,005 \mathrm{~mm}$ para folhas (ABAL, 2004) [6].

Em particular a variante assimétrica da laminação é um processo em que se caracteriza pela passagem do material a ser laminado, por cilindros, os quais proporcionam assimetria ao longo da espessura devido as características destes. Características estas que podem ser: diferença de diâmetro dos cilindros, diferenças de velocidades utilizadas durante o processo ou diferença de atrito. Ao realizar este tipo de laminação, além de uma deformação compressiva, o material também é deformado sob uma condição cisalhante (Zuo, 2008; HALLBERG, 2012) [5] [7].

Conforme ilustração presente na Figura 1, $Y$ corresponde a velocidade e d ao diâmetro do cilindro. Para que ocorra a assimetria no processo, é necessário ao menos que d' seja diferente de d" e/ou $Y^{\prime}$ seja diferente de $Y^{\prime \prime}$.

Lee e Lee (2007) [8] utilizaram em seu estudo o processo de laminação assimétrica em chapas de alumínio AA1050, com intuito de avaliar o refinamento de grão após processos de deformação plástica severa. Para esta análise, a espessura inicial da chapa era de $9 \mathrm{~mm}$, e com deformação de 10\% com lubrificação. Ao final, obteve-se uma chapa com espessura de $4,4 \mathrm{~mm}$. Após laminada, a chapa foi recozida em banho de sal por $1 \mathrm{~h}$ em temperatura de $400^{\circ} \mathrm{C}$. Os autores observaram uma redução do tamanho de grão inicial, que mediam $100 \mu \mathrm{m}$, para tamanho de grão na faixa de $2 \mu \mathrm{m}$ quando realizados tratamentos de recozimento com baixas temperaturas. 


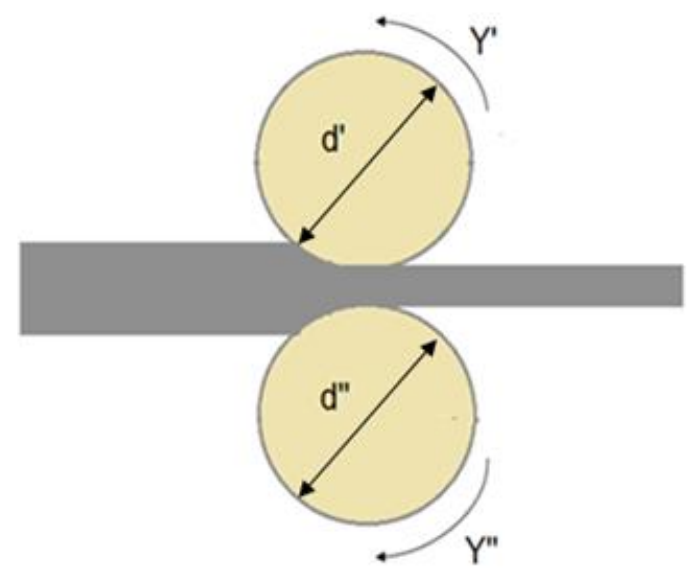

Figura1: llustração do processo de laminação assimétrica

Loorentz (2012) [9] realizou um estudo utilizando a laminação assimétrica para investigar a evolução microestrutural e da propriedade mecânica dureza de uma liga de alumínio 5052 homogeneizada a $823 \mathrm{~K}$ durante 30 minutos. Como parâmetro variante de assimetria, foram utilizados diferentes diâmetros de cilindros de laminação. Foram aplicadas três diferentes velocidades entre cilindros: 1:1, 1:2 e 1:4, ou seja, partiu de uma condição de laminação convencional. Foi estabelecida uma redução de $50 \%$ da espessura, por passe, atingindo uma deformação de 0,8. Como resultado, o autor verificou que com a utilização da condição de laminação convencional (1:1), não foi satisfatória para promoção de um refinamento de grão, quando comparada com as outras duas condições empregadas. No que se refere a dureza, foi verificado que todas as diferentes condições aumentaram esta propriedade em no mínimo, 1,5 vezes do valor da amostra como recebida. Foi encontrado um maior valor de dureza, sendo este de $115 \mathrm{HV}$. Tal medida foi realizada na face superior da chapa em que foi deformada utilizando relação de 1:4 entre cilindros. Em sequência, foi notado um menor incremento de dureza quando utilizada relação de velocidade 1:2. Quando analisada a amostra proveniente da laminação convencional, notou-se o menor aumento de dureza entre todas.

Assim sendo, o presente trabalho tem por objetivo, avaliar por meio da dureza, a evolução microestrutural de amostras submetidas a diferentes processamentos de laminação quando comparadas a amostra como recebida, buscando evidências de encruamento do material.

\section{MATERIAL E MÉTODOS}

Para todos os processamentos e análises, foram utilizados corpos de prova provenientes de uma chapa de alumínio 7475 T7351 laminada a quente, com espessura de $12,5 \mathrm{~mm}$. Esta foi produzida sob condição de tratamento térmico T7351, isto é, tratamento térmico de solubilização, com posterior alívio de tensões por estiramento e endireitamento, seguido de tratamento térmico de superenvelhecimento.

Inicialmente foi realizada análise de espectroscopia de emissão ótica nos laboratórios do CTS- Solda (Centro de Tecnologia SENAI/RJ- Solda) com finalidade de obtenção da composição química da liga objeto de estudo. Encontra-se descrito na tabela 1 o percentual presente de cada elemento na liga. 
Tabela 1. Composição química da liga de alumínio 7475 T7351

\begin{tabular}{lclc}
\hline Elemento & $\%$ & Elemento & $\%$ \\
\hline $\mathrm{Al}$ & 89,5000 & $\mathrm{Ga}$ & 0,0092 \\
\hline $\mathrm{ZN}$ & 5,6700 & $\mathrm{Mn}$ & 0,0090 \\
\hline $\mathrm{Mg}$ & 2,3800 & $\mathrm{Sn}$ & 0,0057 \\
\hline $\mathrm{Cu}$ & 1,7800 & $\mathrm{~Pb}$ & 0,0041 \\
\hline $\mathrm{Fe}$ & 0,2420 & $\mathrm{Cd}$ & 0,0032 \\
\hline $\mathrm{Cr}$ & 0,1970 & $\mathrm{Co}$ & 0,0032 \\
\hline $\mathrm{Si}$ & 0,0438 & $\mathrm{Ca}$ & 0,0028 \\
\hline $\mathrm{Ti}$ & 0,0413 & $\mathrm{Zr}$ & 0,0007 \\
\hline $\mathrm{V}$ & 0,0184 & $\mathrm{Be}$ & 0,0004 \\
\hline $\mathrm{Ni}$ & 0,0132 & Outros & 0,0754 \\
\hline em peso. & & &
\end{tabular}

Como nomenclatura das amostras, foram adotados os seguintes códigos:

CR - Amostra na condição de como recebida

LC - Amostra deformada por meio de Laminação Convencional

LA - Amostra deformada por meio de Laminação Assimétrica

\subsection{Processamento - Laminação}

Para realização dos processos de laminação, tanto convencional quanto assimétrica, foi utilizado o laminador piloto fabricado pela empresa FENN MFG. Co. modelo D51710:1973, que se encontra devidamente instalado no Laboratório de Processamento Mecânico do Instituto Militar de Engenharia (IME).

\section{- Corpos de prova para laminação}

Para o processo de laminação convencional e assimétrica, foram utilizados corpos de prova com dimensões iguais a $100 \times 50 \times 12,5$ (comprimento - direção de laminação $x$ Largura - direção transversal $x$ Espessura - direção normal).

Quando foi realizada a laminação convencional, o laminador operou em configuração duo, com cilindros de trabalho de $133,70 \mathrm{~mm}$ de diâmetro. Para a laminação assimétrica, o laminador teve sua configuração modificada para configuração quádruo, onde foi feito uso de quatro cilindros, sendo dois de trabalho e mais dois de encosto. Nesta última configuração, os cilindros de trabalho mediam diâmetros de 54,8 e 32,8 mm, o que proporcionou uma assimetria com razão 1,67.

Foram realizadas rotas de passes específicas para cada tipo de laminação, e estão apresentadas nas tabelas 2 e 3 referentes a laminação convencional e assimétrica, respectivamente. Vale ressaltar que em ambos os tipos de processos de deformação, objetivou-se a espessura final de $3 \mathrm{~mm}$. 
Tabela 2. Plano de rota de passes para Laminação Convencional.

\begin{tabular}{cc}
\hline Passes & Deformação (\%) \\
\hline $1^{\circ}$ & $10 \%$ \\
\hline $2^{\circ}$ & $10 \%$ \\
\hline $3^{\circ}$ & $10 \%$ \\
\hline $4^{\circ}$ & $10 \%$ \\
\hline $5^{\circ}$ & $10 \%$ \\
\hline $6^{\circ}$ & $10 \%$ \\
\hline $7^{\circ}$ & $10 \%$ \\
\hline $8^{\circ}$ & $10 \%$ \\
\hline $9^{\circ}$ & $10 \%$ \\
\hline $10^{\circ}$ & $10 \%$ \\
\hline $11^{\circ}$ & $10 \%$ \\
\hline $12^{\circ}$ & $10 \%$ \\
\hline $13^{\circ}$ & $10 \%$ \\
\hline
\end{tabular}

Tabela 3. Plano de rota de passes para Laminação Assimétrica

\begin{tabular}{cc}
\hline Passes & Deformação (\%) \\
\hline $1^{\circ}$ & $10 \%$ \\
\hline $2^{\circ}$ & $10 \%$ \\
\hline $3^{\circ}$ & $10 \%$ \\
\hline $4^{\circ}$ & $10 \%$ \\
\hline $5^{\circ}$ & $10 \%$ \\
\hline $6^{\circ}$ & $10 \%$ \\
\hline $7^{\circ}$ & $10 \%$ \\
\hline $8^{\circ}$ & $10 \%$ \\
\hline $9^{\circ}$ & $10 \%$ \\
\hline $10^{\circ}$ & $10 \%$ \\
\hline $11^{\circ}$ & $10 \%$ \\
\hline $12^{\circ}$ & $10 \%$ \\
\hline $13^{\circ}$ & $10 \%$ \\
\hline $14^{\circ}$ & $10 \%$ \\
\hline $15^{\circ}$ & $10 \%$ \\
\hline $16^{\circ}$ & $10 \%$ \\
\hline $17^{\circ}$ & $10 \%$ \\
\hline $18^{\circ}$ & $10 \%$ \\
\hline
\end{tabular}

\subsection{Caracterização - Propriedades Mecânicas}

Visando caracterizar o material como recebido e após processado por meio de conformação mecânica, no que se concerne a dureza (HV clássica e dinâmica), foram realizadas medidas em um ultramicrodurômetro Vickers instrumentado.

\section{- Ultramicrodurômetro Vickers Instrumentado}

Para a realização de medidas de dureza Vickers, se fez uso de um ultramicrodurômetro instrumentado com indentador Vickers, marca Shimadzu, modelo DUH-211S, que se encontra em funcionamento no laboratório Multiusuário de Caracterização de Materiais do Instituto de Ciência Exatas (ICEx) da Universidade Federal Fluminense (UFF), em Volta Redonda, RJ. 
Todos os ensaios foram realizados com carga mínima de 0,2 gf e carga máxima de $50 \mathrm{gf}$, com velocidade de carregamento e de descarregamento de 7,14 gf/s e $20 \mathrm{~s}$ de permanência na carga máxima aplicada. Todas as medidas foram correlacionadas a três espessuras distintas, sendo estas, 1/4, 1/2 e 3/4 na seção longitudinal da direção de laminação.

Para essa análise, foram preparadas amostras das seções transversais associadas a direção de laminação (espessura). O processo de preparo das amostras se deu da seguinte forma:

- Lixamento com lixas de carbeto de silício de 220, 400, 600, 1000, 1200 e 2500 mesh.

- Polimento mecânico fazendo uso de pasta de diamante na sequência de granulometrias: $6 \mu \mathrm{m}, 3 \mu \mathrm{m}$ e $1 \mu \mathrm{m}$.

\section{RESULTADOS E DISCUSSÃO}

A seguir, encontram-se apresentados os resultados comparativos entre as amostras estudadas, provenientes de diferentes condições de laminação, conforme descrito anteriormente em Material e Métodos.

$\mathrm{Na}$ Tabela 4 são apresentados os resultados da propriedade mecânica de dureza, da amostra como recebida, os quais foram obtidos por meio de ultramicrodureza Instrumentada Vickers, referentes a pontos distintos da espessura (1/4, 1/2 e 3/4) ao longo da seção longitudinal da direção de laminação.

\begin{tabular}{lcccccc} 
Tabela 4. Propriedade durezas Vickers - amostra CR \\
\hline \begin{tabular}{lccccccc}
\hline Espessura & HV & Erro & DHV1 & Erro & DHV2 & Erro \\
\hline $1 / 4$ & 152,1 & 5,2 & 41,4 & 1,5 & 200,1 & 15,3 \\
\hline $1 / 2$ & 150,8 & 4,2 & 34,2 & 2,2 & 215,6 & 47,0 \\
\hline $3 / 4$ & 170,2 & 10,3 & 7,5 & 0,1 & 123,1 & 11,0 \\
\hline
\end{tabular}
\end{tabular}

Ao se analisar a dureza Vickers (HV) ao longo da espessura da amostra como recebida, observa-se valores distintos. Este comportamento provavelmente é explicado devido a presença de grãos alongados e espessos intercalados por outro conjunto de grãos menos grosseiros ao longo da espessura do material, conforme pode ser observado na micrografia obtida por microscopia eletrônica de varredura, apresentado na Figura 2, a qual revela a heterogeneidade microestrutural do material. Tal aspecto da microestrutura é supostamente proveniente da rota de processamento, isto é, consequência da laminação a quente de uma liga monofásica com alta energia de falha de empilhamento, a qual não apresenta transformação alotrópica, e ainda, por possuir microestrutura com grãos alongados e grosseiros, demonstra que a deformação a quente final do material apenas permitiu a recuperação dinâmica da estrutura encruada. 


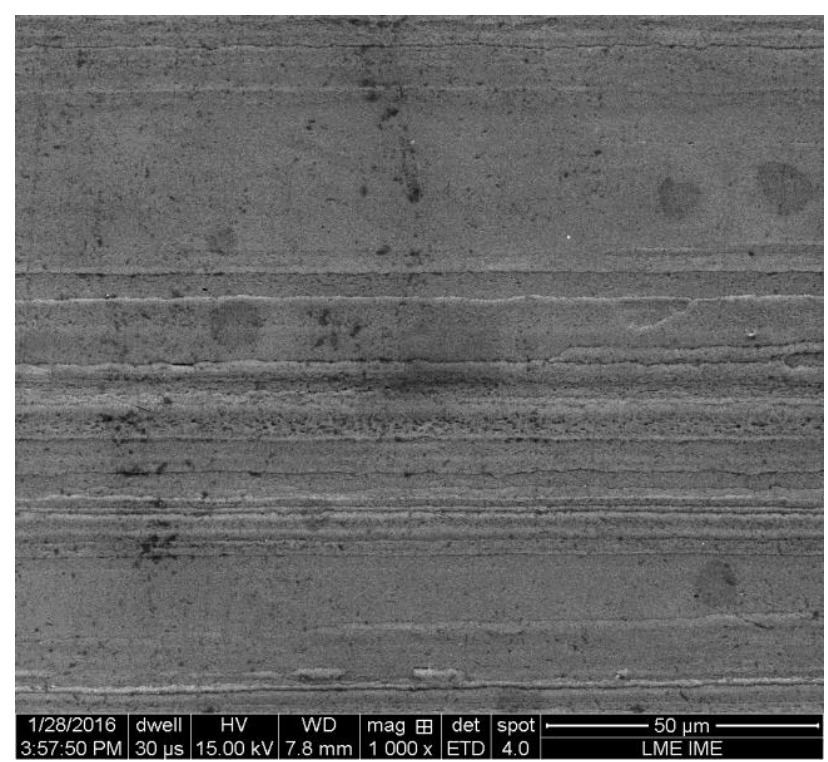

Figura 2. Aspecto microestrutural da amostra na condição de como recebida.

Valores de HV maiores podem assim, estar ligados a grãos menores, indicando desta forma, uma menor dureza para grãos mais grosseiros.

Ao se analisar as durezas dinâmicas Vickers elasto-plástica (DHV1) e plástica (DHV2), presentes na Tabela 4, nota-se valores distintos nos resultados obtidos ao longo da espessura. Podendo ser atribuído a distintos graus de encruamento. Este comportamento também pode ser ocasionado devido a existência de grãos alongados e espessos intercalados por conjuntos de grãos menos espessos ao longo da espessura do material, ligado com o fato dos valores médios das diagonais de indentação serem próximos a $25 \mu \mathrm{m}$, isto é, similar a dimensão de grãos mais espessos. Assim, os valores médios de menor magnitude podem identificar medições realizadas em grãos mais grosseiros, demonstrando que quanto maior 0 grão, menos a resistência a indentação. As amostras aqui comparadas são provenientes de diferentes graus de deformação e rota de processamento conforme descrito anteriormente. Para as amostras LC, foram realizados 13 passes de $10 \%$, totalizando $75 \%$ de deformação acumulada. Para a amostra LA, foram realizados 18 passes (9 passes de $5 \%$ somados a 9 passes de 10\%) totalizando $76 \%$ de deformação acumulada.

$\mathrm{Na}$ Tabela 5 encontram-se descritos os valores médios de durezas Vickers da amostra laminada de forma convencional.

Tabela 5. Propriedade durezas Vickers - amostra LC

\begin{tabular}{lcccccc}
\hline Espessura & HV & Erro & DHV1 & Erro & DHV2 & Erro \\
\hline $1 / 4$ & 171,7 & 3,9 & 121,0 & 2,6 & 345,5 & 50,0 \\
\hline $1 / 2$ & 182,3 & 4,6 & 117,4 & 23,0 & 289,6 & 83,1 \\
\hline $3 / 4$ & 163,4 & 4,6 & 120,7 & 21,0 & 309,3 & 78,3 \\
\hline
\end{tabular}

Para avaliar a homogeneidade, assim como nas outras amostras, foram realizadas medidas ao longo da espessura

Ao verificar os resultados encontrados de HV na amostra LC, é notório que possuem grandezas próximas ao longo de toda espessura. Tal fato indica a presença de uma maior homogeneidade microestrutural do material em comparação com a amostra como recebida (CR). Quando apreciado os valores das durezas DHV1 e DHV2, é visto correlação com um maior erro experimental, que consequentemente está 
ligado intrinsecamente a maior dispersão entre os valores de parâmetros obtidos para cálculo destas propriedades.

Para as amostras laminadas assimetricamente, os resultados médios de durezas Vickers estão apresentados na Tabela 6.

Tabela 6. Propriedade durezas Vickers - amostra LA.

\begin{tabular}{lcccccc}
\hline Espessura & HV & Erro & DHV1 & Erro & DHV2 & Erro \\
\hline $1 / 4$ & 138,2 & 2,4 & 159,6 & 5,0 & 262,8 & 19,8 \\
\hline $1 / 2$ & 136,4 & 2,8 & 149,1 & 2,3 & 225,2 & 4,3 \\
\hline $3 / 4$ & 133,2 & 0,8 & 142,4 & 2,0 & 4216 & 4,0 \\
\hline
\end{tabular}

Ao analisar os valores destas propriedades obtidos, percebe-se que estes estão ainda mais próximos quando comparados aos da amostra como recebida. Assim, resultados tão próximos ao longo da espessura, podem indicar que este tipo de deformação plástica severa inferiu a amostra uma microestrutura ainda mais homogênea.

Outro fator importante a ser notado é a presença da redução do erro experimental. Esta pode ser uma indicação que a microestrutura está mais uniformemente distribuída na direção de laminação, mesmo quando o esperado era o oposto, ou seja, grande variação de características microestruturais devido a presença da assimetria imposta pelo processo entre os dois lados que estiveram em contato com os diferentes cilindros.

Ao comparar as durezas dinâmicas de todas as amostras, percebe-se DHV2 maior que DHV1, tal comportamento esta ligado por valores de $\mathrm{hr}$ (profundidade de indentação para um retorno elástico teórico) serem sempre menores que Hmáx (profundidade máxima), pelo motivo deste último medir a resistência da indentação que está diretamente relacionada com a deformação elástica e plástica do material até o momento em que se atinge força máxima aplicada.

Tendo em vista que para a amostra LA os valores médios das durezas dinâmicas (DHV1 e DHV2) são superiores as demais amostras, não é notado o mesmo em HV (que possui média máxima em LC). Ao se atentar para a dispersão de erros para as amostras LC e LA, especialmente para DHV2, nota-se que os valores de LA se aproximam da faixa inferior da dispersão de LC. Assim, tem-se comportamento de DHV2 similar a HV.

Assim, é esperado que a microestrutura mais homogênea tenha sido causada com o aumento no grau de encruamento da amostra. De modo a poder-se ter resultados mais precisos quanto a evolução estrutural das amostras em estudo, estão sendo conduzidas análises via microscopia eletrônica de varredura em um MEV/FEG para melhor detalhamento microestrutural ao longo da espessura das amostras em estudo.

\section{CONCLUSÃO}

- A amostra LC possui uma maior homogeneidade microestrutural quando comparada com a amostra como recebida (CR).

- A amostra LA apresentou, possivelmente, uma maior homogeneidade microestrutural quando comparada com as outras amostras, indo contra o esperado.

- A diferenciação do percentual de redução por passe, contribuiu supostamente, para uma maior homogeneização da amostra LA. 


\section{Agradecimentos}

A CAPES pela bolsa de mestrado do aluno Andrey Casanova (2014-2016) e doutorado do aluno Andrey Casanova (2017 até o presente) e Saulo Diniz (2014 até o presente). Ao Instituto Militar de Engenharia por viabilizar a pesquisa com toda infraestrutura. Ao prof. Ladário da Silva que possibilitou o uso do equipamento de ultramicrodureza instrumentada, do Laboratório Multiusuário de Caracterização de Materiais do Instituto de Ciências Exatas (ICEx) da Universidade Federal Fluminense de Volta Redonda - RJ. Aos laboratórios do CTS- Solda (Centro de Tecnologia SENAI/RJ- Solda) pela análise de espectroscopia de emissão ótica.

\section{REFERÊNCIAS}

1 ASM Handook. Properties and Selection: Nonferrous Alloys and Special-Purpose Materials. Ohio: ASM International; 1990.

2 Nieh T.G, Wadsworth J, Sherby O.D. Superplasticity in Metals and Ceramics. Nova York: Cambridge University Press; 1997.

3 Nieh T.G, Wadsworth J, Shelby OD. Superplasticity in Metals and Ceramics. Segunda Edição. Nova York: Cambridge University Press; 2005.

4 Valiev R.Z, Korznikov A.V, Mulyukov R.R. Structure and Properties of Ultrafine-Grained Materials Produced by Severe Plastic Deformation. Materials Science and Engineering. 1993.141-148.

5 Zuo F. - Qing et al. Shear Deformation and Grain Refinement in Pure Al by Asymmetric Rolling.Transactions of Nonferrous Metals Society of China. 2008.18:774-777.

6 Fundamentos e Aplicações do Alumínio. São Paulo: Associação Brasileira do Alumínio (ABAL); 2004.

7 Hallberg $\mathrm{H}$. Influence of Process Parameters on Grain Refinement in AA1050 Aluminum During Cold Rolling. International Journal of Mechanical Sciences. 2012;66:260-272.

8 Lee J.K, Lee D.N. Texture Control and Grain Refinement of AA1050 Al Alloy Sheets by Asymmetric Rolling. International Journal of Mechanical Sciences. 2008. 50: 869-887.

9 Loorentz, Y.G.KO. Microstructure Evolution and Mechanical Properties of Severely Deformed Al Alloy Processed by Differential Speed Rolling. Journal of Alloys and Compounds, School of Materials Science and Engineering. 2011. 712-749 